\title{
Agriculture driving male expansion in Neolithic Time
}

\author{
Chuan-Chao Wang, Yunzhi Huang, Xue'er Yu, Chun Chen, Li Jin \& Hui Li* \\ MOE Key Laboratory of Contemporary Anthropology, School of Life Sciences, Fudan University, Shanghai 200438, China
}

Received January 13, 2016; accepted February 3, 2016; published online April 28, 2016

Citation: Wang, C.C., Huang, Y., Yu, X., Chen, C., Jin, L., and Li, H. (2016). Agriculture driving male expansion in Neolithic Time. Sci China Life Sci 59, 643-646. doi: 10.1007/s11427-016-5057-y

\section{Dear Editor,}

Dispute between agriculture and initial population expansion in Neolithic Time has been in suspense for a long time. Agriculture first appeared in the Fertile Crescent of West Asia about 11-12 thousand years ago (kya), with domesticating few wild plant and animal species. There is also evidence of the cultivation of rice and millet in Yangtze and Yellow River Basins of China approximately 9 kya. During the subsequent times between 9 and 4 kya, agriculture arose independently in multiple regions except Europe. Because of food production can support far higher population densities compared to hunting and foraging, major population expansions has long been suggested to has begun after the invention of agriculture (Diamond, 2002; Jobling et al., 2004). However, genetic evidence from maternal mitochondrial genomes suggests major population expansions began before the emergence of agriculture. Therefore, role of agriculture that played in initial population expansions still remains controversial. There are also numerous studies on population expansion using paternal Y chromosome single nucleotide polymorphism (SNPs) in the genealogical tree and rapidly mutating short tandem repeats (STRs) (Wang and Li, 2013). However, nonrandom sampling of SNPs can result in an ascertainment bias, and to choose whether the evolutionary rate or the genealogical rate of STRs in Y chromosome dating is controversial, since the result can be almost three-fold difference (Wang and Li, 2013). With the advent of next-generation sequencing technology, 1000 Genomes Project has sequenced whole Y

*Corresponding author (email: LHCA@ @udan.edu.cn) chromosomes from more than 500 males (1000 Genomes Project Consortium, 2012), which provides a wonderful chance to estimate population sizes through time from a set of globally distributed populations without ascertainment bias and helps to solve the long discuss.

We analyzed about 8.9 mega-base pairs on the unique regions of $\mathrm{Y}$ chromosome and whole mitochondrial genomes of 526 male individuals from three African, five $\mathrm{Eu}-$ ropean, three Asian and three American populations sequenced in the 1000 Genome Project (Table S1 in Supporting Information) (1000 Genomes Project Consortium, 2012) (see Materials and methods online for details).

A maximum likelihood tree was constructed using $\mathrm{Y}$ chromosomal SNPs (Figure 1a, Figure S1 in Supporting Information). Containing samples from haplogroup A, B, C, $\mathrm{D}, \mathrm{E}, \mathrm{G}, \mathrm{I}, \mathrm{J}, \mathrm{N}, \mathrm{O}, \mathrm{Q}, \mathrm{R}$, and T, the tree is a good representation of geographical paternal lineages. To infer the expansion time, we calculated the date of each divergence event throughout the tree using Bayesian method (Van Geystelen et al., 2013; Guindon et al., 2003) with a constant mutation rate of $1 \times 10^{-9}$ substitution/baseyear (Drummond et al., 2002). Most of the main haplogroups (C, I, J, O, E and R) emerged between 25-30 kya right before LGM. Almost all the primary sub-clades of main haplogroups branched off after LGM and before Neolithic Time (10-15 kya). The long internal branch lengths within those primary sub-branches indicate low population growth and frequent bottlenecks in the late Upper Paleolithic Age. However, Neolithic Time saw the great booming of new emerging lineages (Figure 1a, Figure S1 in Supporting Information). We have also identified many star-like structures in the phylogenetic tree, such as I1a, O2b, O3a2c1 (O $\alpha$ and $\mathrm{O} \beta), \mathrm{O} 3 \mathrm{a} 1 \mathrm{c1}(\mathrm{O} \gamma)$, 


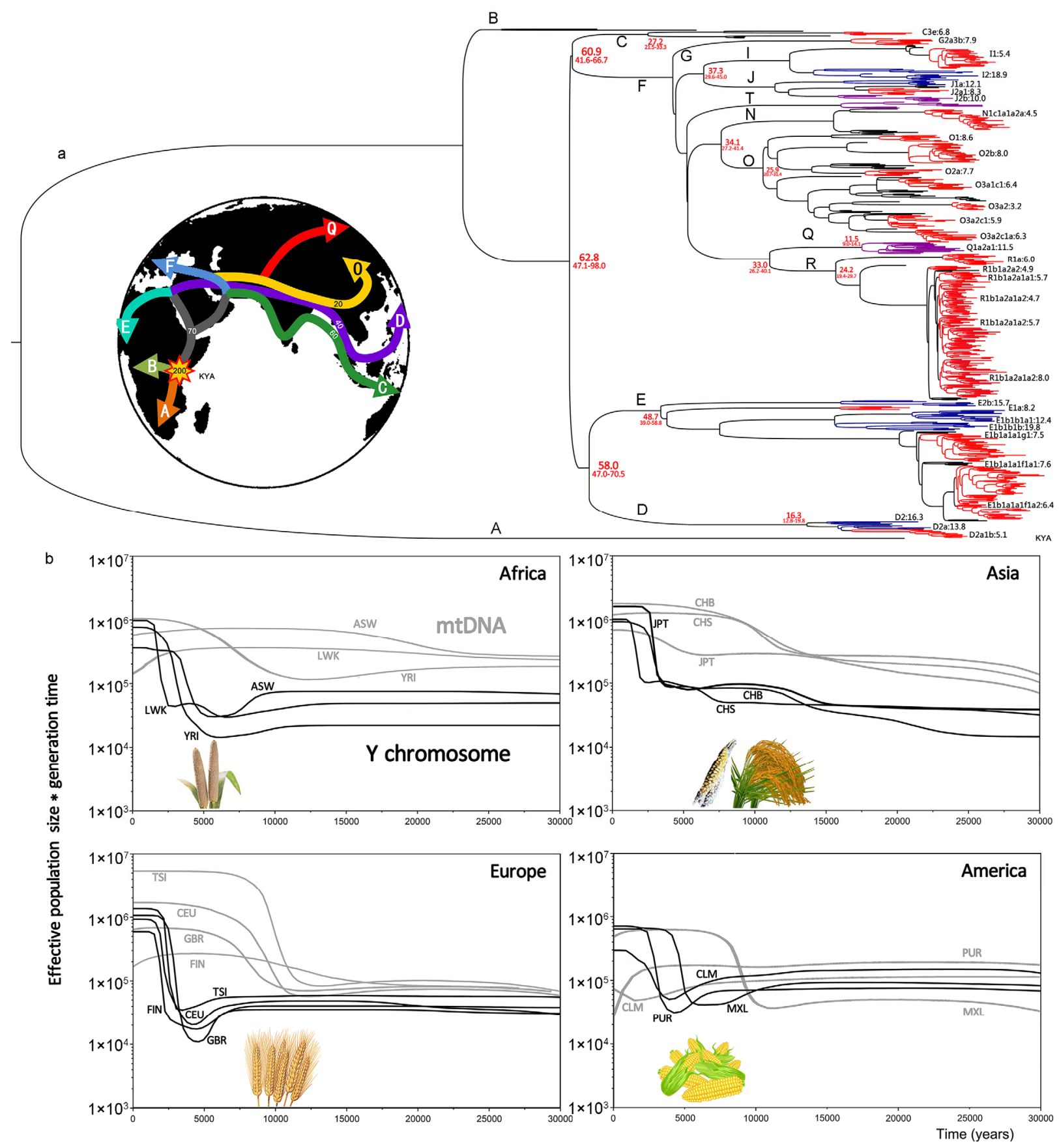

Figure 1 Demonstrating the expansions of $Y$ chromosomes. a, Phylogenetic tree of human Y chromosome. This tree was constructed using 526 samples sequenced in 1000 Genomes Project. The branch lengths are proportional to the number of SNPs on the branch. Numbers in red indicate the coalescence time (in years) and 95\% confidence intervals of the node. For more details, see Figure S1 in Supporting Information. b, Bayesian skyline plots of Y chromosome and mtDNA showing the effective population sizes of male and female through time. The $y$-axis is the product of effective size and generation time. The $x$-axis is the time from present in units of years. IBS was not included in this figure for its small sample size. Detailed settings refer to Figure S2 in Supporting Information. ASW, people with African ancestry in Southwest United States. LWK, Luhya in Webuye, Kenya. YRI, Yoruba in Ibadan, Nigeria. CEU, Utah residents with ancestry from Northern and Western Europe. FIN, Finnish in Finland. GBR, British from England and Scotland, UK. IBS, Iberian populations in Spain. TSI, Toscani in Italia. CHB, Han Chinese from Beijing Normal University, China. CHS, Han Chinese in Hunan and Fujian Provinces, China. JPT, Japanese in Tokyo, Japan. CLM, Colombians in Medellin, Colombia. MXL, people with Mexican ancestry in Los Angeles, California. PUR, Puerto Ricans in Puerto Rico.

R1a, R1b1a2a1, E1b1a1a1g, and E1b1a1a1f1a (Figure 1a, Figure S1 in Supporting Information). The short internal branch lengths within the star-like clades relative to the other haplogroups are interpreted as a strong signal of recent rapid population expansions of those clades. All the star-like lineages also coalesced in Neolithic Time (5-8 kya). The Neolithic expansions for R1b, O $\alpha \beta \gamma$ have also been suggested in recent published whole $\mathrm{Y}$ chromo- 
some sequencing papers (Poznik et al., 2013; Yan et al., 2014). Those Neolithic expanding clades comprise a large proportion of current population. For instance, R1b1a2a1 is the dominant paternal lineage of Western Europe, accounting for more than half of the population (Drummond and Rambaut, 2007). O $\alpha, \mathrm{O} \beta$, and $\mathrm{O} \gamma$ reach frequencies of $40 \%$ in Chinese (Yan et al., 2014; Darriba et al., 2012). O2b comprises more than $20 \%$ of Japanese and Korean (Yang, 2007). E1b1a1a1g and E1b1a1a1f1a account for over $80 \%$ of population in many parts of West Africa, Central Africa, East Africa as well as Southern Africa (Fan and Yao, 2013). Thus, the time estimation of lineage coalescence showed that paternal population growth could be mainly attributed to Neolithic expansion.

We then estimated effective population size through time via coalescent Bayesian Skyline plots (Van Geystelen et al., 2013; Guindon et al., 2003) to infer population size changes during the LGM and the advent of agriculture (Figure 1b). The plots all show evidence of population growth and also reveal clear differences in male and female population histories. We infer rapid, roughly exponential population growth in most European and Asian maternal lineages right after the LGM (12-8 kya), followed by a long period of very slow growth (since 8-7 kya). YRI in Africa and MXL in America also show the similar pattern as European and Asian populations. However, at the paternal side, all the populations show the most pronounced expansion from 6.5 to $2 \mathrm{kya}$, with a 10 - to 100 -fold increase in population size. Except CHB and JPT had a slight growth at about 13 kya, the initial male expansion of global populations all started in Neolithic Time rather than just after LGM. The estimated timing of this male growth phase fits perfectly with archaeological evidence for the advent of agriculture in each region (Table S2 in Supporting Information). We then calculated the rate of population growth per year (Gignoux et al., 2011). The fastest population growth for most European, Asian and MXL maternal lineages occurred about 9-11 kya (Figure 1b, Table S2 in Supporting Information), corresponding to the initiation of a stable, global warming period after LGM. We also found a sharp increase in maternal population size at 33-35 kya in Asian populations, which might reflect the population explosion after initial peopling of this continent. However, the fastest growth intervals for global male populations range from 1.5-3.3 kya, which are 1-3 kya later than the advent of agriculture (Figure 1b, Table S2 in Supporting Information). During this period, agriculture became mature and intensive, and can sustain a rapidly growing population. In addition, all skyline plots except Asian population (Figure 1b) showed obvious bottlenecks during the agricultural transition, which might be the impact of male-dominated demic diffusion of agriculture.

To summarize, the correspondence between the coalescence age of most paternal lineages and the population growing periods observed in skyline plots suggested the initial male population expansion began within the Neolithic Time, probably due to the advent and spread of agriculture. Agriculture has provided a much more stable food supply than hunting and foraging, leading to higher population fertility and infant survival rate. More importantly, as agriculture has kept male away from dangerous hunting, the reduction in hunting-related mortality of males might contribute most to this sex-biased Neolithic expansion. It is essential to determine precise mutation rate for reliable time estimation in this research. Currently available Y chromosome mutation rate comes from the divergence time of human and chimpanzees, sequencing results of deep-rooting pedigrees and calibration based on founding migration events. We previously used data from 1000 Genomes Project and archaeological dating of anatomically modern humans $(\mathrm{AMH})$ out of Africa corresponding to time to the common ancestor of $\mathrm{Y}$ chromosome haplogroup $\mathrm{C}$ and $\mathrm{F}$ to select the most proper mutation rate. It proves that mutation rate from deep-rooting pedigrees with reference of dating of human entering America is reliable (Wang et al., 2014), thus we use mutation rate on the order of $1.0 \times 10^{-9}$ mutations/(nucleotide generation) from deep-rooting pedigrees for this research. In addition, 1000 Genome Project has updated data Phase 3 (1000 Genomes Project Consortium, 2015), subsequent analysis ulteriorly verified our result.

Compliance and ethics The author(s) declare that they have no conflict of interest.

Acknowledgements This work was supported by the National Excellent Youth Science Foundation of China (31222030), National Natural Science Foundation of China (91131002), MOE Scientific Research Project (113022A), and Shanghai Shuguang Project (14SG05).

Darriba, D., Taboada, G.L., Doallo, R., and Posada, D. (2012). jModelTest 2: more models, new heuristics and parallel computing. Nat Methods 9, $772-772$.

Diamond, J. (2002). Evolution, consequences and future of plant and animal domestication. Nature 418, 700-707.

Drummond, A.J., Nicholls, G.K., Rodrigo, A.G., and Solomon, W. (2002). Estimating mutation parameters, population history and genealogy simultaneously from temporally spaced sequence data. Genetics 161, 1307-1320.

Drummond, A.J., and Rambaut, A. (2007). BEAST: Bayesian evolutionary analysis by sampling trees. BMC Evol Biol 7, 214.

Fan, L., and Yao, Y. (2013). An update to MitoTool: using a new scoring system for faster mtDNA haplogroup determination. Mitochondrion 13, 360-363.

Genomes Project Consortium, Auton, A., Brooks, L.D., Durbin, R.M., Garrison, E.P., Kang, H.M., Korbel, J.O., Marchini, J.L., McCarthy, S., McVean, G.A., and Abecasis, G.R. (2015). A global reference for human genetic variation. Nature 526, 68-74.

Gignoux, C.R., Henn, B.M., and Mountain, J.L. (2011). Rapid, global demographic expansions after the origins of agriculture. Proc Natl Acad Sci USA 108, 6044-6049.

Guindon, S., and Gascuel, O. (2003). A simple, fast, and accurate algorithm to estimate large phylogenies by maximum likelihood. Syst Biol 52, 696-704.

Jobling, M., Hollox, E., Kivisild, T., Tyler-Smith, C. (2004). Agricultural expansions. In: Human Evolutionary Genetics. (New York: Garland 
Publishing), pp. 299-306.

Pinhasi, R., Fort, J., and Ammerman, A.J. (2005). Tracing the origin and spread of agriculture in Europe. PLoS Biol 3, 2220-2228.

Poznik, G.D., Henn, B.M., Yee, M.C., Sliwerska, E., Euskirchen, G.M., Lin, A.A., Snyder, M., Quintana-Murci, L., Kidd, J.M., Underhill, P.A., and Bustamante, C.D. (2013). Sequencing Y chromosomes resolves discrepancy in time to common ancestor of males versus females. Science 341, 562-565.

The 1000 Genomes Project Consortium (2012). An integrated map of genetic variation from 1,092 human genomes. Nature 491, 56-65.

Van Geystelen, A., Decorte, R., and Larmuseau, M.H.D. (2013).
AMY-tree: an algorithm to use whole genome SNP calling for Y chromosomal phylogenetic applications. BMC Genomics 14, 101.

Wang, C., Gilbert, M.T., Jin L., and Li, H. (2014). Evaluating the Y chromosomal timescale in human demographic and lineage dating. Investig Genet 5, 12 .

Wang, C., and Li, H. (2013). Inferring human history in East Asia from Y chromosomes. Investig Genet 4, 11.

Yan, S., Wang, C., Zheng, H., Wang, W., Qin, Z., Wei, L., Wang, Y., Pan, X., Fu, W., He, Y., Xiong, L., Jin, W., Li, S., An, Y., Li, H., and Jin, L. (2014). Y chromosomes of $40 \%$ Chinese are descendants of three Neolithic super-grandfathers. PLoS One 9, e105691

Open Access This article is distributed under the terms of the Creative Commons Attribution License which permits any use, distribution, and reproduction in any medium, provided the original author(s) and source are credited.

\section{SUPPORTING INFORMATION}

Figure S1 Phylogenetic tree of human Y chromosome with time estimation for each clade.

Figure S2 Bayesian Skyline Plots of effective population size through time with population details.

Table S1 Sample information and haplogroup assignment

Table S2 Population growth rates calculated from skyline plots

The supporting information is available online at life.scichina.com and link.springer.com. The supporting materials are published as submitted, without typesetting or editing. The responsibility for scientific accuracy and content remains entirely with the authors. 